\title{
Dynamics of Line Defects in Nematic Liquid Crystals
}

\author{
P. E. Cladis, W. van Saarloos, P. L. Finn, and A. R. Kortan \\ AT\& T Bell Laboratories, Murray Hill, New Jersey 07974 \\ (Received 16 October 1986)
}

\begin{abstract}
An experiment is performed in which a topological line defect $\left(S=-\frac{1}{2}\right)$ is forced to move with constant speed $c$ under the action of an applied voltage $V$. We argue that the line speed is determined by a competition between the viscous damping and the free energy that the system gains by displacing the line, so that $c=\beta V$, with $\beta=(1 / 2 \gamma b)\left(\varepsilon_{a} K / \pi\right)^{1 / 2}$ and $b$ a number $\sim 1$ determined only by viscous effects close to the core of the defect.
\end{abstract}

PACS numbers: $61.30 . J f, 47.35 .+\mathrm{i}, 62.30 .+\mathrm{d}$

In the search for a fundamental understanding of the dynamics of nonlinear systems, there has recently been considerable interest in the dynamics of nematic liquid crystals. $^{1,2}$ Nematic liquid crystals are the simplest of the liquid-crystal phases and a great deal is already known about their static and dynamic behavior, well described by nonlinear partial differential equations, particularly in the limit of small deviations from equilibrium. ${ }^{3}$

One reason for studying nonlinear dynamical properties is that domain walls in liquid crystals ${ }^{4}$ are often described by nonlinear equations reminiscent of the sineGordon equation, but with the important difference that they are first order in time instead of second order. This is because of the large viscous damping. For equations of this type, the recently developed marginal stability theory ${ }^{5}$ predicts the propagation speed of a domain wall separating a stable and an unstable state. Since electric ${ }^{2}$ and/or magnetic ${ }^{4}$ fields can be used to create such states in liquid crystals, domain walls of this type can be studied experimentally. In agreement with theory, ${ }^{6}$ their speed is determined by a balance of the viscous damping, the electrostatic energy difference between domains, and the elastic energy of the wall.

Nematic defects are macroscopic objects; so direct observations of their behavior can be made simply with use of an optical polarizing microscope. They are topological in character and can be created under controlled conditions in the laboratory but their dynamics have received only limited attention. ${ }^{7}$ In the absence of external fields, the elastic deformation induced by a defect falls off slowly (inversely proportional to the distance from the defect), and the "interaction" energy between defects ${ }^{7}$ or between a defect and a wall ${ }^{3}$ therefore depends logarithmically on the distance. As a result, the annihilation time of two $S=+1$ defects is proportional to the square of the initial separation. ${ }^{7}$ To study defect dynamics without the influence of walls, the induced deformations have to be localized in a region far from boundaries. This can be done with the aid of external fields. In the experiment described in this paper we create a line defect whose excess energy is linearly proportional to its distance from one of the walls; we study its motion and show that it rapidly approaches a constant speed, as expected on the basis of elementary considerations. Although the propagation of a line is physically different from domain walls, its speed is again determined by the competition between the free energy gained by displacing the line and viscous damping hindering its motion. ${ }^{8}$ The problem is analogous to that of a solid body falling through a viscous medium in a constant force field.

Figure 1(a) shows the experimental setup. The nematic liquid crystal 5CB (pentylcyanobiphenyl) is sandwiched between two transparent InO electrodes separated by wire spacers. The wires are electrically insulated from the InO surfaces by a thin coating of varnish. All the surfaces are treated so that the director $\mathbf{n}$,
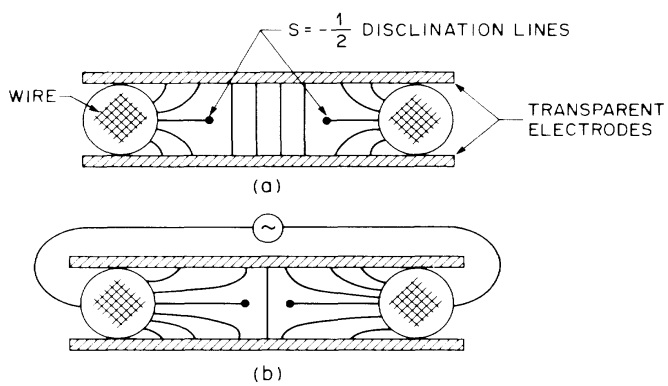

(b)
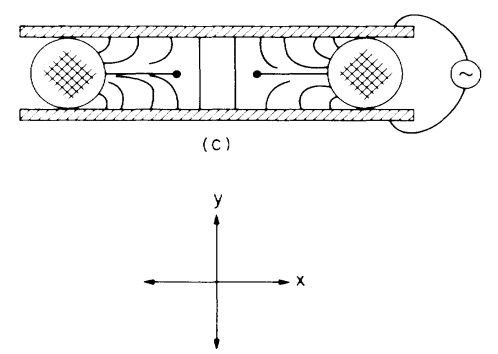

FIG. 1. (a) The experimental geometry; $V_{h}=V_{v}=0$. (b) The left line moves to the right when $V_{h}$ is turned on. (c) The director pattern after the first reorientation wave has swept through immediately after $V_{v}$ is turned on. The director configuration is metastable to the left of the line and stable to the right of the line. The line moves to the left. 
the preferred direction along which the liquid-crystal molecules tend to align, is perpendicular to them. ${ }^{3}$ These boundary conditions force the director to form $S=-\frac{1}{2}$ line defects parallel to the wires in the midplane of the sample. The sample thickness $2 h$ is $140 \mu \mathrm{m}$, determined by the wire thickness, and the distance between the two wires is $\sim 1 \mathrm{~mm}$. The experiment is to apply a $1-\mathrm{kHz}$ ac voltage, $V_{h}=90 \mathrm{~V}$, between the two wires forcing the line to move away from the wire [Fig. 1(b)] since the dielectric anisotropy $\varepsilon_{a}$ of $5 \mathrm{CB}$ is positive, $\varepsilon_{a} \sim 10$. Eventually the line comes to rest a distance $l$ from the wire. $V_{h}$ is then turned off and simultaneously (within $50 \mu \mathrm{sec}$ ), a vertical voltage, $V_{v}$, is applied to the InO electrodes forcing the line to move back towards the wire $(l \rightarrow 0)$ with a speed $c$. The experiment is filmed with a video camera and the motion of the line analyzed.

As soon as $V_{v}$ is turned on a wave of director reorientation moves through the material without displacing the line. We interpret this as a readjustment of the director pattern, shown in Fig. 1(c), to one in which the vertical distortion is now localized in the middle of the sample. After this, the line defects start to move towards the wire. The first reorientation wave is fast $^{9}$ (milliseconds) compared to the motion of the line defect (seconds).

The importance of this first wave is that it sets up a stable but more energetic director configuration on the wire side of each line. Physically, if the center of the line is at $y=0$, then for $y>0$ the director wants to turn one way and for $y<0$ the other way. For concreteness, consider the left defect. The only way the less energetic configuration can replace the more energetic splay-bend distortion of the director is by movement of the line to the left. While the region to the right is absolutely stable,${ }^{10}$ the region to the left is metastable. The line defect can thus be viewed as an energy barrier that has to be overcome before the total energy can be reduced by replacing the metastable state with the absolutely stable state. The physics is therefore different from that of the motion of a wall that separates an unstable state from a stable state, discussed in the introduction.

Contrary to the case of the interaction of two defects in the absence of fields, the electric field in this experiment confines the distortion of the director to a thin region around $y=0$. This results in an excess energy for the line that is linear in the distance $l$ from the wall, and implies a constant force on the line. As discussed in more detail below, we then expect the line speed to approach a constant value $c$ whose voltage dependence is determined by the change in excess energy with voltage. This is borne out by the experiments.

A few millseconds after the application of $V_{v}$, the line starts to move towards the wire. We track it over a distance of about $200 \mu \mathrm{m}$ from the beginning of its motion until it comes to rest close to the wire. Figure 2(a) shows the variation of $c$ with applied voltage at $32^{\circ} \mathrm{C}$. Except for the region $V \sim 0, c$ is proportional to the ap- (a)

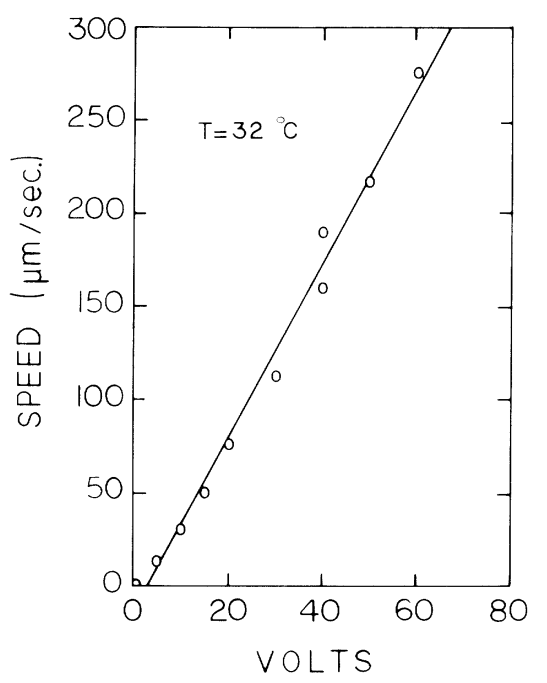

(b)

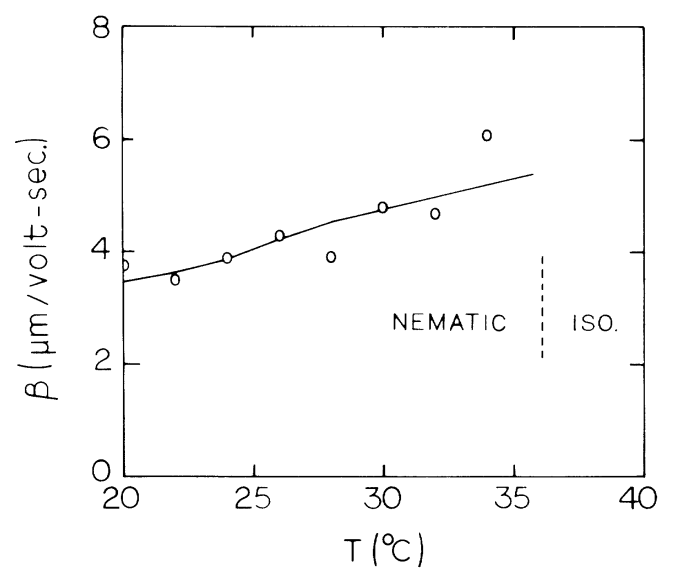

FIG. 2. Characteristics of the line motion. (a) Line speed vs the applied vertical field showing that the speed is linear in the applied voltage. The slope of this line is $\beta$. (b) $\beta$ vs temperature for $5 \mathrm{CB}$. The nematic to isotropic transition temperature for $5 \mathrm{CB}$ is $36^{\circ} \mathrm{C}$.

plied voltage. A linear least-squares fit to the data gives the constant of proportionality as $\beta=4.7 \mu \mathrm{m} / \mathrm{V} \mathrm{sec}$. Figure 2(b) shows $\beta$ as a function of temperature. The data are shown as points.

Using the definition of the coordinate axis shown in Fig. 1, and calling $\theta$ the angle between $\mathbf{n}$ and the $x$ axis, we then have for the free energy in the one-constant approximation

$$
F=\frac{1}{2} \int d V\left\{K\left[\left(\frac{\partial \theta}{\partial x}\right)^{2}+\left(\frac{\partial \theta}{\partial y}\right)^{2}\right]-\frac{\varepsilon_{a}}{4 \pi} E^{2} \sin ^{2} \theta\right\}
$$

The fundamental length scale $\xi$ set by the competition of the elastic and electric forces is $\xi=\left(4 \pi K / \varepsilon_{a} E^{2}\right)^{1 / 2}$. From this, we get $\xi / 2 h=0.22 / V_{v}$ in $5 \mathrm{CB}$, so that for $V_{v}$ 
larger than a few volts the free-energy density is nonzero only in a region thin compared with the sample thickness. For distances much larger than $\xi$ to the left of the defect $(\partial \theta / \partial x)^{2}$ is negligible and since $\theta=\pi / 2$ in the undistorted state to the right of the line, the region to the left has for $l \gg \xi$ an excess free energy per unit line length,

$$
F_{\text {exc }}=l \int_{-h}^{+h} d y\left[\frac{1}{2} K(\partial \theta / \partial y)^{2}-\left(\varepsilon_{a} / 8 \pi\right) E^{2}\left(\sin ^{2} \theta-1\right)\right]+F_{\text {core }}
$$

Here $l$ is the distance between the wire and the defect line and $F_{\text {core }}$ is the free energy of the core region within a distance $\xi$ from the line defect. To evaluate the first term in $F_{\text {exc }}$ we first express $\partial \theta / \partial y$ in terms of $\sin ^{2} \theta$. The equation away from the disclination is

$$
K \partial^{2} \theta / \partial y^{2}=-(d / d \theta)\left(\varepsilon_{a} E^{2} / 8 \pi\right) \sin ^{2} \theta .
$$

A first integral of this equation can be obtained easily. To see this note, that (2) is like the equation of motion for a particle in a potential. Here, the analog of energy conservation of the particle translates to constancy of the sum of the elastic and electric energy density in (1), i.e.

$$
\frac{1}{2} K(\partial \theta / \partial y)^{2}+\left(\varepsilon_{a} / 8 \pi\right) E^{2} \sin ^{2} \theta=\text { const . }
$$

On the boundaries, $\theta= \pm \pi / 2$ and $\partial \theta / \partial y$ is negligible for $h \gg \xi$, hence const $=\varepsilon_{a} E^{2} / 8 \pi$. Equation (3) then finally yields for $F_{\text {exc }}$

$$
\begin{aligned}
F_{\text {exc }} & =F_{\text {core }}+2 l \int_{-h}^{+h} d y \frac{\varepsilon_{a}}{8 \pi} E^{2} \cos ^{2} \theta \\
& =F_{\text {core }}+l\left(\varepsilon_{a} K / \pi\right)^{1 / 2} E,
\end{aligned}
$$

where the integral was evaluated by transformation to $\theta$ as the integration variable, with use of $d x=\xi d \theta /|\cos \theta|$.

The driving force for this motion is $-d F_{\text {exc }} / d l$, while the energy is dissipated only in a small region of radius of order $\xi$ around the defect line. Thus, on dimensional grounds, we expect an equation for its motion of the form

$$
2 b \gamma_{\mathrm{eff}} d l / d t=-d F_{\mathrm{exc}} / d l=-E\left(\varepsilon_{a} K / \pi\right)^{1 / 2},
$$

implying that the velocity ${ }^{11} c=-d l / d t$ is

$$
c=\left(E / 2 b \gamma_{\mathrm{eff}}\right)\left(\varepsilon_{a} K / \pi\right)^{1 / 2} \text {. }
$$

In these equations $\gamma_{\mathrm{eff}}$ is an effective viscosity and $b$ a number of order unity. The term on the left-hand side represents the friction that occurs in a region of size $\xi$ near the defect where the director changes rapidly in time. The number $b$ depends only on the details of the energy dissipation in this region, where the strain is singular. As a result, $b$, which in principle has to be determined by solving of the full hydrodynamic equations for the two-dimensional flow around the defect, will depend on the core size and may show some weak field dependence as well. A rough estimate (expected to be an overestimate ${ }^{12}$ ) for $b$, obtained by approximation of the structure near the defect by that of the defect in the absence of fields, gives $b \approx 2.1$ for field strengths used in our experiments. Because the strain near the defect consists of both splay and bend, we take $\gamma_{\text {eff }}=\frac{1}{2}\left(\gamma_{\text {bend }}\right.$ $\left.+\gamma_{\text {splay }}\right)$, where ${ }^{3} \gamma_{\text {splay }} \sim \gamma_{1}$, the orientation viscosity, but $\gamma_{\text {bend }}=\gamma_{1}-\alpha_{2}^{2} /\left(\eta_{b}-\gamma_{2}\right)$ because of backflow effects. As mentioned earlier, the terms $\gamma_{\text {eff }}$ and $b$ on the left-hand side of Eq. (5) both depend on the precise structure near the core, such as the splay and/or bend character of the strain and the core size. Since such details are not known with great accuracy, the above estimates are crude, and our approximation for $\gamma_{\text {eff }}$ should be considered correct to only about $20 \%$.

The data of Fig. 2(a) give $\beta=c / V_{v}=4.7 \mu \mathrm{m} / \mathrm{V} \mathrm{sec}$, with an error of about $5 \%$ estimated from the scatter in the data. For $5 \mathrm{CB}$ at $30^{\circ} \mathrm{C},{ }^{13} \gamma_{1}=0.50 \mathrm{dyn} / \mathrm{cm}^{2} \mathrm{sec}$, $\left(K_{1}+K_{3}\right) / 2=5.5 \times 10^{-7}$ dyn, $\varepsilon_{a}=9.8, \eta_{b}=0.25$ poise, and $\gamma_{2}=-0.56$ poise. With these values, Eq. (6) pre$\operatorname{dicts} \beta=c / V \approx 4.2 b^{-1} \mu \mathrm{m} / \mathrm{V}$ sec. Comparison with the experimentally obtained value then gives $b \approx 0.9$, slightly more than a factor of 2 smaller than our crude estimate, suggesting that the core drags some material with it to reduce the director rotations. Figure 2(b) compares the experimentally observed temperature dependence of $\beta$ with the temperature dependence as predicted with the aid of Eq. (6) with fixed $b$. In both cases, the temperature dependence appears to be qualitatively correct.

While we interpret the data as support for our basic notion that the director pattern simply translates in the applied field with a speed determined by a balance of the viscous force against the elastic and electrostatic energies, a more stringent comparison of theory and experiment requires a closer examination of backflow effects (taken into account here in an approximate way by picking an effective viscosity that is the average of the pure splay and bend viscosities ${ }^{3}$ ), and of the dissipation and possibly drag in the small region around the core. Since in our experiment the driving force of the motion is known accurately (even when the splay and bend elastic constants are not the same ${ }^{14}$ ) while the dissipation is determined only by the details of the core structure, we hope that this will provide a way to study small-scale properties of defects through their dynamics. ${ }^{15}$

In conclusion, we have studied the motion of a line defect when its driving force is precisely known and independent of the position of the defect. The resulting line speed is accounted for by a simple balance between the change in free energy gained by displacing the line and the energy dissipation near the defect. The experiment therefore opens up the possibility to study viscous effects near the highly strained core region.

\footnotetext{
${ }^{1}$ Zhu Guozhen, Phys. Rev. Lett. 49, 1332 (1982).
} 
${ }^{2}$ P. E. Cladis, H. R. Brand, and P. L. Finn, Phys. Rev. A 28, 512 (1983).

3P. G. de Gennes, The Physics of Liquid Crystals (Oxford Univ. Press, Oxford, 1974).

${ }^{4}$ W. Helfrich, Phys. Rev. Lett. 21, 1518 (1968); P. G. de Gennes, J. Phys. (Paris) 32, 789 (1971); F. Brochard, J. Phys. (Paris) 33, 607 (1972); L. Leger, Solid State Commun. 10, 697 (1972), and Mol. Cryst. Liq. Cryst. 24, 33 (1973).

${ }^{5}$ G. Dee and J. S. Langer, Phys. Rev. Lett. 50, 383 (1983); E. Ben-Jacob, H. Brand, G. Dee, L. Kramer, and J. S. Langer, Physica (Amsterdam) 14D, 348 (1985); W. van Saarloos, to be published.

${ }^{6}$ See for an analysis of Leger's experiments (Ref. 4) X. Y. Wang, Phys. Lett. 112A, 402 (1985), and Phys. Rev. A 32, 3126 (1985). For a discussion of Zhu's experiment (Ref. 1) see X. Y. Wang, Phys. Lett. 98A, 259 (1983), and Commun. Theor. Phys. 2, 1307 (1983), and Lin Lei, Shu Changqing, and Xu Gang, J. Stat. Phys. 39, 633 (1985). A discussion of the experiments of Ref. 2 will be given elsewhere.

${ }^{7}$ Yu A. Dreizin and A. M. Dykhne, Zh. Eksp. Teor. Fiz. 61, 2140 (1972) [Sov. Phys. JETP 34, 1140 (1972)]; A. S. Sonin, A. N. Chuvyrov, A. A. Sobachkin, and V. L. Ovchinnikov, Fiz. Tverd. Tela (Leningrad) 18, 3099 (1976) [Sov. Phys. Solid State 18, 1805 (1976)].

${ }^{8}$ This idea goes back to the work of Brochard, Ref. 4.

${ }^{9}$ Elastic forces do not play a significant role in this initial reorientation that takes place in a time of order $\gamma / \varepsilon_{a} E^{2}$ with $\gamma$ the appropriate combination of viscosities damping the splaybend deformation. In the regime studied here, this is about a few milliseconds agreeing with our experimental observations.

${ }^{10} \mathrm{This}$ picture is supported by a more detailed stability analysis. To the left of the line defect, the director approaches the $x$-indpendent profile $\theta_{0}(y)$ given by Eq. (2). On our substituting $\theta=\theta_{0}(y)+\Delta \theta(y) e^{-\omega t+k x}(K>0)$ and linearizing, $\Delta \theta$ is found to obey an equation of Schrödinger type with $\omega$ playing the role of the energy eigenvalue. With the use of arguments similar to those discussed by A. C. Scott, F. Y. F. Chu, and D. W. McLaughlin, Proc. IEEE 61, 1443 (1973), it can then be shown that $\omega>0$, so the region to the left of the line is linearly stable.

${ }^{11}$ In Ref. 9 the annihilation of two defects in the absence of fields is studied. Here, $F_{\text {exc }} \sim \ln (l)$ and Eq. (5) gives $l^{2}$ $\sim\left(t-t_{0}\right)$.

${ }^{12}$ The energy dissipation per unit time and line length is according to Ref. $3 \int \gamma(\partial \theta / \partial t)^{2} d x d y=c^{2} \gamma \int(\partial \theta / \partial x)^{2} d x d y$. Equating this to the change in elastic energy per unit time $\left(=c d F_{\text {exc }} / d l\right)$ yields $b=\frac{1}{2} \int(\partial \theta / \partial x)^{2} d x d y$. The use of the expression for $\theta$ near a $-\frac{1}{2}$ defect, $\theta=-\phi / 2$, gives

$$
b=\frac{1}{2} \int_{r_{c}}^{\xi} d r \int d \phi\left(\sin ^{2} \phi / 4 r\right)=\frac{1}{8} \pi \ln \left(\xi / r_{c}\right),
$$

where $r_{c}$ is the core size. From the formula after Eq. (1a) we get $\xi \sim 6000 \AA$ for $V=50 \mathrm{~V}$; taking $r_{c}=28 \AA$, we then get $b \sim 2.1$. We expect this to be an overestimate since the effect of the electric field will be to reduce $(\partial \theta / \partial x)^{2}$ in the expression for $b$, and since the core size may be a few times the molecular size.

${ }^{13}$ K. Skarp, S. Lagerwall, and B. Stebler, Mol. Cryst. Liq. Cryst. 60, 215 (1980).

${ }^{14}$ The field $\theta(y)$ in (1b) away from the defect is a combination of splay and bend; the analysis leading to (4) can be extended to the case $K_{1} \neq K_{3}$. For $5 \mathrm{CB}$, the resulting correction is about $3 \%$.

${ }^{15}$ In this regard, we note that in Ref. 12 a larger value of the core size would give a lower estimate of $b$, e.g., $r_{c}=60 \AA$, gives $b \approx 1.8$. 